\title{
El acortamiento de los isquiosurales
}

\author{
Shortening of the Hamstring Muscles \\ MIGUEL VIDAL BARBIER \\ Departamento de Educación Física \\ IES Honori García. La Vall d'Uixó (Castellón)
}

TANIA VIDAL ALMIÑANA

Departamento de Ciencias Experimentales

IES Caparrella (Lleida)

MERCEDES ALMELA ZAMORANO

Departamento de Psicobiología

Universitat de València

\author{
MIGUEL VIDAL ALMIÑANA \\ Departamento de Educación Física \\ IES Ulldecona (Tarragona)
}

\author{
Correspondencia con autor \\ Miguel Vidal Barbier \\ miguelvidalbarbier@gmail.com
}

\section{Resumen}

El acortamiento de los isquiosurales, según varios autores (Bado, 1977; Jordà, 1971; Lambrinudi, 1934; Latorre \& Hernández, 2003; López, Ferragut, Alacid, Yuste, \& García, 2008; Santonja, Andújar, \& Martínez, 1994; Santonja, Ferrer, \& Martínez, 1995), puede producir alteraciones en la estática del raquis que pueden desencadenar anomalías en su estructura y procesos álgidos. Estos hechos hacen que la detección temprana de un acortamiento en estos músculos sea importante para prevenir posibles trastornos. Nosotros encontramos una disminución en el nivel de flexibilidad de los isquiosurales-raquis medida a través del test del cubo o Distancia Dedos Planta (D-D-P), de 4,2 cm de los alumnos que habían cursado sus estudios en nuestro centro en el año 2007, con respecto a la flexibilidad de los alumnos de la misma edad que cursaron sus estudios en el año 1991, achacando tal pérdida a la disminución en la actividad física que se realiza en la actualidad (Vidal, Vidal, \& Almela, 2009). Pero no conocíamos si esta merma de la flexibilidad afectaba al incremento del número de casos de cortedad isquiosural. Para conocer si, además de la disminución de flexibilidad, se producía un incremento en el número de casos con cortedad isquiosural, hicimos una valoración-comparación del número de alumnos que padecían cortedad isquiosural en el año 1986 con respecto a los alumnos que la padecían en el año 2008. Encontramos un incremento en el número de casos, pasando de un 5,13\% en 1986 a un $20 \%$ en 2008. Por tanto, la disminución de actividad física que se está produciendo en nuestros jóvenes, sobre todo por el incremento del tiempo que dedican a las nuevas tecnologías de la información y comunicación (TIC), no sólo puede repercutir negativamente en la merma de flexibilidad sino también lo hace en el incremento de sujetos con cortedad isquiosural.

Palabras clave: incremento acortamiento isquiosural, disminución actividad física

\begin{abstract}
Shortening of the Hamstring Muscles

The shortening of the hamstring muscles, according to various authors (Bado, 1977; Jordà, 1971; Lambrinudi, 1934; Latorre \& Hernández, 2003; López, Ferragut, Alacid, Yuste, \& García, 2008; Santonja, Andújar, \& Martínez, 1994; Santonja, Ferrer, \& Martinez, 1995), can produce alterations in the static of the spinal column which can cause anomalies in its structure and crucial processes. These facts mean that early detection of a shortening of these muscles is important in the prevention of possible disorders. We found a reduction in the level of flexibility of the hamstring muscles-spine measured by the cube test or Finger-Floor Distance (F-F-D) of $4.2 \mathrm{~cm}$ in students who had studied in our school in 2007, with respect to the flexibility of students of the same age who were studying in 1991, attributing this loss to the reduction in physical activity which is typical at present (Vidal, Vidal, \& Almela, 2009). But we did not know whether this shrinkage in flexibility affected the increase in the number of cases of shortened hamstring muscles. To know whether, in addition to the reduction of flexibility, it produced an increase in the number of cases of short hamstring muscles, we made a comparative evaluation of the number of students who had short hamstring muscles in 1986 as against the students who suffered it in 2008 . We found an increase in the number of cases, moving from $5.13 \%$ in 1986 to $20 \%$ in 2008 . Therefore, the reduction in physical activity which is taking place in our young people, above all through the increase in time devoted to the new information and communication technology (ICT), could not only have negative repercussions in lesser flexibility but also in the increase of subjects with short hamstring muscles.
\end{abstract}

Keywords: increase in short hamstring muscles, reduction in physical activity 


\section{Introducción}

Casi con toda certeza, el Ardipithecus ramidos, que representa nuestro ancestro evolutivo, no tenía problemas de espalda ya que sus desplazamientos los realizaba a cuatro patas. Tras la adopción de la posición erecta, hace unos cinco millones de años, el Australopithecus obtuvo muchas ventajas ya que, al estar más alto, podía detectar antes tanto a sus presas como a sus enemigos, también le permitía refrigerarse mejor y economizar reservas energéticas en sus largos desplazamientos y, sobre todo, pudo manipular objetos al liberar sus manos. Pero no todo iban a ser ventajas, tuvimos que pagar un alto precio por todas estas mejoras, precio que seguimos pagando y que cada vez parece ser más elevado. Este pago a realizar se refleja en poseer una parte de nuestra estructura ósea susceptible de problemas y es nuestro raquis el que debe pagar la adopción de esta nueva posición. Si bien las curvas fisiológicas: cifosis y lordosis, según nos indica Kapandji (1977), nos confieren una mayor resistencia a las cargas axiales (ya que la resistencia de una columna es proporcional al cuadrado del número de curvaturas más uno), la alteración (incrementar o disminuir) del grado de estas curvaturas interfiere negativamente en su mecánica, acarreando posibles dolencias. Una alteración que puede afectar negativamente a nuestro raquis es el acortamiento isquiosural (semitendinoso, semimembranoso que se insertan en la tibia y bíceps femoral que se inserta en el peroné), que puede producir, en primera instancia, una retroversión de la pelvis (Latorre \& Hernández, 2003) al tener un punto de anclaje sobre la misma que tira de ella. Esta retroversión puede afectar a la columna lumbar disminuyendo su curvatura y, como medida compensatoria, se puede producir un incremento de la curvatura cifótica torácica (Butler \& Chir ,1955; Jordà, 1971). Al respecto, ya en 1964, Bado, Barros, Ruggiero y Navita afirmaron que los síndromes de isquiosurales cortos se acompañan de incremento de cifosis (en Santonja et al., 1994). Además, hay autores que achacan a esta cortedad problemas de lumbalgias, espondilolisis, espondilolistesis (Barash et al., 1970; Phalen y Dickson 1961; citados por Santonja et al., 1995), enfermedad de Scheuermann, incluso hernias discales de la región lumbosacra (Santonja et al., 1994).

Estas posibles alteraciones sobre la columna que pueden producir un acortamiento isquiosural, se ven potenciadas cuando esta se moviliza de forma reiterada en flexión, ya que la tensión de los isquiosurales sobre la pelvis disminuye su posibilidad de basculación (anteversión) en flexión, lo que conlleva, por parte del sujeto que realiza la flexión, en un intento de llegar más lejos, un incremento de la presión sobre la parte anterior de los cuerpos vertebrales, propiciando el acuñamiento de las mismas; también se facilita la aparición de herniaciones intraesponjosas. Bado (1977) demuestra la importancia de la limitación de la flexión de la coxofemoral, la cual limita la de la pelvis y esta la de la columna sacrolumbar y lumbar, por lo que se establecerán movimientos compensadores (aumento de la movilidad del sector dorso-lumbar), que, al establecerse desde los primeros años de la vida y mantenerse a lo largo del crecimiento, dará lugar a una mayor amplitud de los movimientos compensatorios y a una modificación estructural del raquis o dorso curvo. Bado et al., unos años antes, en 1964 demuestran la relación estadística con el dorso curvo, asegurando que todo dorso curvo se debe a una "retracción" de esta musculatura, aunque no todas las "retracciones" de isquiosurales se acompañan de una hipercifosis.

Esta relación entre cortedad isquiosural y afecciones del raquis Micheli (1979) la explica por el crecimiento más rápido producido en los huesos que en los ligamentos y tendones durante el segundo estirón del crecimiento.

La invasión de las nuevas tecnologías de la información y comunicación (TIC), ha cambiado las actuaciones de las personas que tienen a su alcance estos medios, afectando sobre todo a la vida de los jóvenes, disminuyendo el tiempo de actividad física que estos realizan diariamente, cambiándolo por tiempo de inactividad.

Un estudio hecho en 2004 por la Confederación Española de Amas de Casa, Consumidores y Usuarios (en Moreno y Charro, 2007), revela que los/as niños/as españoles/as ven la televisión una media de 3,6 horas/día. Otro estudio en adolescentes españoles de 14 a 18 años sobre el consumo de medios de comunicación (en Moreno \& Charro, 2007) realizado en el 2003 demuestra que el $76 \%$ tenía radiocasete en la habitación; el $67 \%$ reproductor de CD, el $57 \%$ ordenador, el $52 \%$ televisión y conexión a Internet y el $38 \%$ videoconsola, nos dice que el tiempo dedicado a las TIC es de 4,5 h/día. Si a estas $4,5 \mathrm{~h} /$ día de estar sentado y por tanto de no actividad física, le sumamos 5-7 horas de clase, más 1 hora de repaso (música, inglés, etc.), más 1,5 horas de las comidas, más 9 horas de dormir, nos suman 21-23 horas de reposo casi absoluto, por tanto quedan solo 1-3 horas para poder realizar actividad física.

Por tanto, la cantidad de actividad física que se realiza en la actualidad por parte de los jóvenes se ha visto mermada y sustituida por actividades pasivas que, posiblemente, resulten en una merma de la condición física de nuestros jóvenes. Así los informes del programa Perseo (Ministerio de Sanidad y Consumo) indican el desalentador dato de: 


\begin{tabular}{|c|c|c|c|c|c|c|c|c|c|c|c|c|}
\hline & \multicolumn{6}{|c|}{ Alumnos año 1986} & \multicolumn{6}{|c|}{ Alumnos año 2008} \\
\hline & \multicolumn{2}{|c|}{$\begin{array}{c}\text { 1. }^{\circ} \text { Bachiller } \\
14 \text { años }\end{array}$} & \multicolumn{2}{|c|}{$\begin{array}{c}2 .^{\circ} \text { Bachiller } \\
15 \text { años }\end{array}$} & \multicolumn{2}{|c|}{$\begin{array}{c}\text { 3. }{ }^{\circ} \text { Bachiller } \\
16 \text { años }\end{array}$} & \multicolumn{2}{|c|}{$\begin{array}{l}1^{\circ} \text { ESO } \\
14 \text { años }\end{array}$} & \multicolumn{2}{|c|}{$\begin{array}{l}2 .^{\circ} \text { ESO } \\
15 \text { años }\end{array}$} & \multicolumn{2}{|c|}{$\begin{array}{c}\text { 1. }{ }^{\circ} \text { Bachiller } \\
16 \text { años }\end{array}$} \\
\hline & Chicas & Chicos & Chicas & Chicos & Chicas & Chicos & Chicas & Chicos & Chicas & Chicos & Chicas & Chicos \\
\hline$n$ & 110 & 73 & 71 & 62 & 73 & 59 & 70 & 66 & 51 & 49 & 60 & 49 \\
\hline
\end{tabular}

\section{Tabla 1}

Número de alumnos por cursos en el Tiempo A (1986) y Tiempo B (2008)

dos de cada tres niños en edad escolar tienen una baja actividad fisica extraescolar; practican menos de una hora al día de cualquier tipo de actividad fisica.

En un estudio que realizamos en 2009 (Vidal et al.) en el que, entre otras cualidades físicas, comparamos el nivel de flexibilidad que tenían los sujetos de 16 años en el año 1991 frente a sujetos de la misma edad del año 2007, comprobamos una pérdida de flexibilidad en este corto periodo de tiempo de tan solo 16 años (pero que los primeros no se vieron influidos por las TIC y los segundos sí que lo estuvieron) de 4,2 cm (pérdida estadísticamente significativa). Nuestra pregunta tras esta constatación fue: ise han incrementado el número de alumnos con cortedad isquiosural tras la introducción de las TIC? Para dar respuesta a esta pregunta realizamos el siguiente trabajo.

\section{Método}

Tomamos los datos del test de flexibilidad de Distancia Dedos Planta (D-D-P) de los alumnos de 14, de 15 y 16 años que cursaban $1 .^{\circ}, 2 .^{\circ}$ y $3 .^{\circ}$ de bachiller en el año 1986 (periodo A) y los comparamos con datos de los alumnos de la misma edad que en el año 2008 (periodo B) cur-

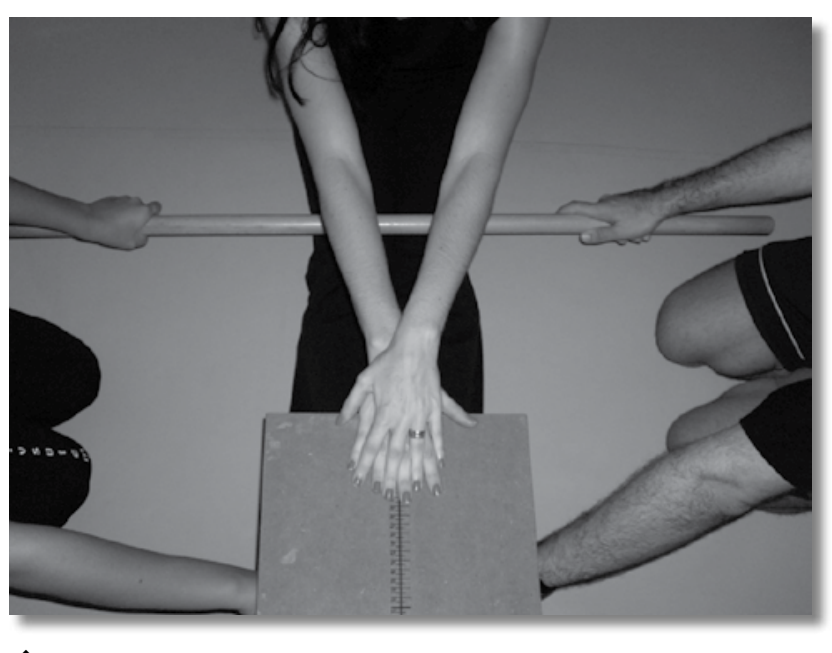

Figura 1

Valoración del test Distancia Dedos Planta saban $3 .^{\circ} \mathrm{ESO}, 4 .^{\circ}$ ESO y $1 .^{\circ}$ de bachillerato, el número de alumnos de cada grupo aparece reflejado en la tabla 1 .

La valoración del test de Distancia Dedos Planta (D-D-P) lo realizamos (fig. 1): el alumno, sentado en el suelo con las piernas estiradas, coloca los pies juntos sobre la base de un cubo de madera, con los dedos de las manos entrelazados y el extremo de estos a la misma altura, efectúa una flexión de tronco llevando las manos lo más lejos posible. Para incrementar la fiabilidad de la medida, dos ayudantes presionan sobre la punta de los pies (que están apoyados sobre la base del cubo) del examinado para que los mantenga juntos, así mismo, se presiona sobre sus piernas (por encima de la rótula) con una pica para evitar que doble las rodillas.

Reglas: 1) no doblar las rodillas; 2) no dar tirones sino progresar lentamente tocando la madera; 3) mantener la posición de máxima flexión dos segundos.

Previo a la realización del test (siempre se realizaron por la mañana) se efectuaba un calentamiento de unos 8-10 minutos consistente en: tres minutos de carrera continua, dos-tres minutos de distintos tipos de carrera (elevando rodillas, talones al culo, con rotación de brazos, etc.), tras lo cual se pasaba a realizar estiramientos, finalizando con 4 series de treinta segundos de estiramiento específico de isquiosurales.

Es importante señalar que el profesor que impartió las clases de Educación Física (y realizó la toma de datos) en ambos periodos temporales, fue el mismo, aplicando un sistema de trabajo semejante en ambos grupos, lo cual elimina la posible influencia que un distinto tipo de trabajo pudiera producir en los resultados del test.

Este test básicamente nos ofrece una medida del nivel de estiramiento de los isquiosurales y de los extensores del tronco. Este es un test de medida longitudinal que, aún siendo menos discriminatorio para determinar el grado de extensibilidad de la musculatura isquiosual que los llamados tests de recorrido angular, como puede ser el test de "elevación de pierna recta", es muy sensible para ofrecer un análisis del comportamiento de la columna vertebral durante la flexión forzada del 
tronco, además, su reproductibilidad es muy alta, es muy fácil de aplicar, y requiere poco material, lo cual hace que sea ampliamente utilizado en el mundo escolar y deportivo (Santonja et al., 1995). Por otra parte, hay que señalar que su validez, según López, Ferragut, Alacid, Yuste y García (2008) es moderada para los chicos y algo mayor par las chicas. Kippers y Parker (1987) señalan que la participación del movimiento vertebral en la flexión completa de cadera y tronco, arroja resultados no significativos en relación con la distancia dedos-planta, por lo que a máxima flexión de tronco la distancia lineal alcanzada supone principalmente una medición de la capacidad de extensibilidad de la musculatura isquiosural. Rodríguez et al. (1999) indican que el principal problema que tiene este test en su valoración es que no tiene en cuenta las medidas antropométicas de los sujetos que valora, así el poseer unos largos brazos o viceversa influirá en la medida de forma positiva o negativa.

Aunque los datos de normalidad a nivel de isquiosurales no están adecuadamente definidos, pues existe una gran variabilidad según edades, género, niveles de ac- tividad, nosotros para este trabajo nos basaremos en el baremo que establece Ferrer en su tesis doctoral (1998):

- Normalidad $\rightarrow \geq-2 \mathrm{~cm}$

- Cortedad grado I $\rightarrow$ entre $<-2 \mathrm{y}>-10$

- Cortedad grado II $\rightarrow \leq-10 \mathrm{~cm}$

Por tanto, consideraremos que existe acortamiento isquiosural cuando la medida en el test (D-D-P) sea inferior a $-2 \mathrm{~cm}$.

\section{Resultados}

En nuestro estudio se produjo una disminución de la flexibilidad entre el tiempo A (1986) y el tiempo B (2008) (ver tabla 2). El género no influyó en la disminución de la flexibilidad (todas las $p>0,05$ ), por lo que esta se produjo de forma similar en chicos y chicas (ver tabla 3 y fig. 2). Estos resultados confirman los datos de nuestro estudio anterior (Vidal et al., 2009), en el que detectamos una disminución en el nivel de flexibilidad en el transcurso de tan solo 16 años.

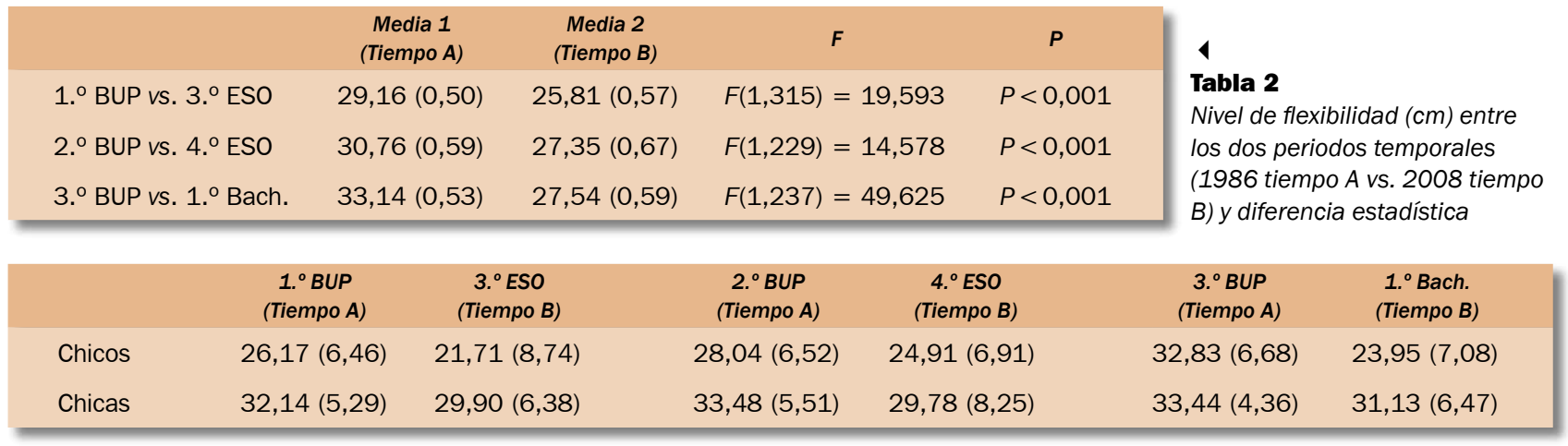

$\Delta$

Tabla 3

Promedio del nivel de flexibilidad y desviación típica en chicos y chicas en el tiempo A (1986) y tiempo B (2008)

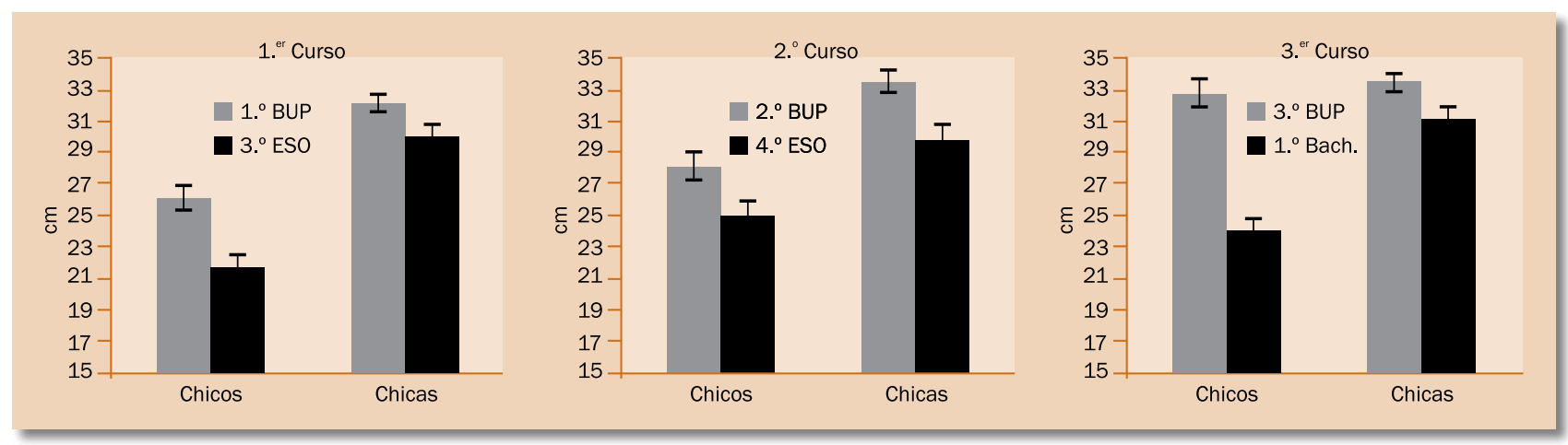

Figura 2

Promedio del nivel de flexibilidad en chicos y chicas en el tiempo A (gris) y tiempo B (negro) 


\begin{tabular}{|c|c|c|c|c|c|c|c|c|c|c|c|c|}
\hline & \multicolumn{6}{|c|}{ Alumnos año 1986} & \multicolumn{6}{|c|}{ Alumnos año 2008} \\
\hline & \multicolumn{2}{|c|}{$\begin{array}{l}1^{\circ} \text { BUP } \\
14 \text { años }\end{array}$} & \multicolumn{2}{|c|}{$\begin{array}{l}2 .^{\circ} \mathrm{BUP} \\
15 \text { años }\end{array}$} & \multicolumn{2}{|c|}{$\begin{array}{l}3 .^{\circ} \text { BUP } \\
16 \text { años }\end{array}$} & \multicolumn{2}{|c|}{$\begin{array}{l}1^{\circ} \text { ESO } \\
14 \text { años }\end{array}$} & \multicolumn{2}{|c|}{$\begin{array}{l}2 .^{\circ} \text { ESO } \\
15 \text { años }\end{array}$} & \multicolumn{2}{|c|}{$\begin{array}{c}1 .^{\circ} \text { Bachiller } \\
16 \text { años }\end{array}$} \\
\hline & Chicas & Chicos & Chicas & Chicos & Chicas & Chicos & Chicas & Chicos & Chicas & Chicos & Chicas & Chicos \\
\hline$n$ & 0 & 12 & 1 & 9 & 0 & 1 & 4 & 29 & 5 & 11 & 2 & 18 \\
\hline$\%$ & 0 & 16,4 & 1,4 & 14,5 & 0 & 1,69 & 5,71 & 43,93 & 9,8 & 22,44 & 3,33 & 36,73 \\
\hline
\end{tabular}

Tabla 4

Número de alumnos con cortedad isquiosural y porcentaje según baremo de Ferrer

\begin{tabular}{|c|c|c|c|c|c|c|}
\hline & \multicolumn{3}{|c|}{ Valores de flexibilidad alumnos año 1986} & \multicolumn{3}{|c|}{ Valores de flexibilidad alumnos año 2008} \\
\hline & Chicas & Chicos & Chicas+Chicos & Chicas & Chicos & Chicas+Chicos \\
\hline$n$ & 254 & 194 & 448 & 181 & 164 & 345 \\
\hline N. ${ }^{\circ}$ de alumnos con cortedad & 1 & 22 & 23 & 11 & 58 & 69 \\
\hline$\%$ & 0,39 & 11,34 & 5,13 & 6,83 & 32,04 & 20 \\
\hline
\end{tabular}

$\triangle$

Tabla 5

Número y porcentaje de acortamientos entre Tiempo A (1986) y Tiempo B (2008)

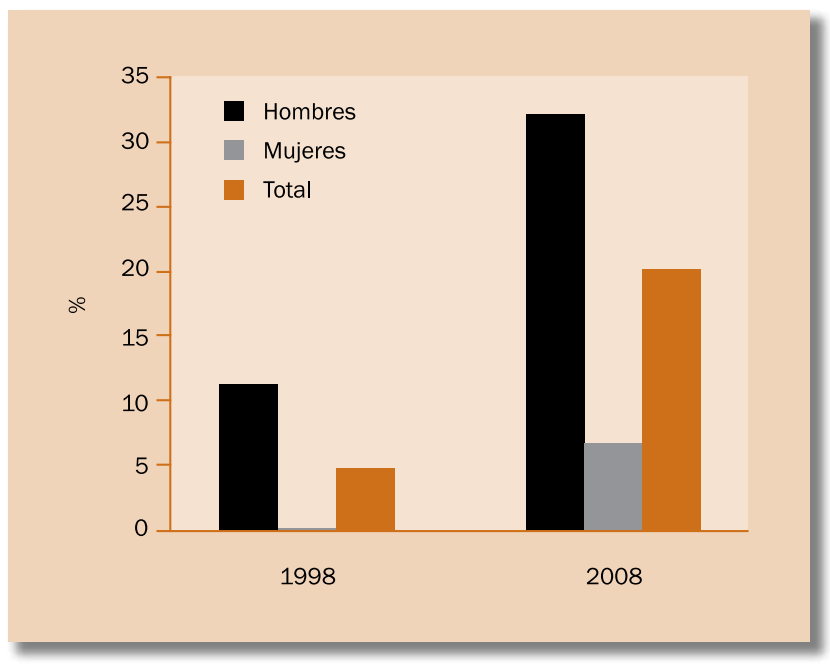

Figura 3

Porcentaje de acortamientos en el Tiempo A (1986) y Tiempo B (2008)

El género no influyó en la disminución de la flexibilidad entre el tiempo A y el tiempo B, puesto que el efecto fue similar en los chicos y en las chicas. Por lo tanto, se confirman los datos de nuestro estudio anterior (Vidal et al., 2009), en el que detectamos una disminución en el nivel de flexibilidad en el transcurso de tan sólo 16 años.

Tras aplicar el baremo establecido por Ferrer (1998) (cortedad isquiosural cuando la distancia alcanzada es
$<-2 \mathrm{~cm}$ ), encontramos un número creciente de acortamientos isquiosurales al comparar el número de casos existentes en el año 1986 con respecto a 2008, tal y como se refleja en la tabla 4.

Cuando los datos los valoramos teniendo en cuenta solamente el género y globalmente (tabla 5 y fig. 3), se ve más clara la relación creciente del número de acortamientos que se han producido en estos 22 años transcurridos, pasando cuando se tiene en cuenta a toda la muestra de 23 casos de acortamiento a 69 casos, que supone un incremento de casi un $15 \%$ de casos.

Estos datos nos reflejan dos aspectos muy evidentes:

1. En las chicas se dan muchos menos casos de cortedad isquiosural que en los chicos en ambos periodos de tiempo, así en el primer periodo valorado (1986), aparece 1 chica frente a 22 chicos, que supone un $0,39 \%$ frente al $11,34 \%$, y en el segundo periodo, en 2008, aparecen 11 chicas frente a 58 chicos, que supone un $6,83 \%$ de las chicas y un $32,04 \%$ en los chicos.

2. El aumento de casos de cortedad se ha incrementado muchísimo en tan sólo 22 años, así se ha pasado de 23 sujetos (chicos más chicas) con cortedad que supone el $5,13 \%$ a 69 alumnos con acortamiento, que representa el $20 \%$ de la población testeada. 
Cuando estos datos los sometemos al análisis estadístico log-lineal de tres factores (tiempo: $1986 \mathrm{vs}$. 2008; género: hombre vs. mujer; cortedad: sí vs. no), la interacción entre los tres factores no fue significativa, $\chi^{2}(1)=2.067, p=0,151$, lo que indica que el género de los participantes no determinó el cambio en los valores de cortedad a través del tiempo. El modelo final estuvo compuesto por las interacciones de tiempo $\times$ cortedad y cortedad $\times$ género. La bondad de ajuste de este modelo fue $\chi^{2}(2)=2.777, p=0,249$. La interacción entre Tiempo y Cortedad refleja que la probabilidad de tener cortedad era 5 veces superior en 2008 que en 1986 $\left(\chi^{2}(1)=42.001, p<0,001\right)$. La interacción entre cortedad y género $\left(\chi^{2}(1)=73.470, p<0,001\right)$ refleja que la probabilidad de los chicos de tener cortedad sin tener en cuenta el tiempo (A vs. B) era 9,6 veces superior que en el caso de las chicas.

\section{Discusión}

En primer lugar, los datos encontrados reafirman el hallazgo de nuestro primer estudio (Vidal et al., 2009) y nos indican que el nivel de flexibilidad ha disminuido tanto en chicos como en chicas desde el periodo $\mathrm{A}$ al periodo $\mathrm{B}$.

En este trabajo hemos encontrado un claro incremento de los casos de cortedad isquiosural, pasando de un $5,13 \%$ en el año 1986 a un $20 \%$ en el año 2008, siendo significativa esta diferencia. Puede ser responsable de este importante incremento de casos de cortedad el cambio de vida que se ha impuesto en estos últimos años, haciendo que se reduzca muchísimo el tiempo dedicado a la actividad física y, en consecuencia se haya incrementado el tiempo de inactividad. Hass, Feigenbaum y Franklin (2001) señalan que entre los niños y adolescentes de 6 a 17 años de edad, menos de la mitad se ejercitan a un nivel considerado suficiente para alcanzar beneficios para la salud y la aptitud física.

Los datos acerca de la prevalencia de cortedad isquiosural que nos ofrece la literatura son muy variados e influyen en la disparidad de los mismos tanto la edad de los sujetos testeados como el test utilizado y el criterio de cortedad aplicado. Ya Bado, en 1964, en su estudio sobre alrededor de 800 niños escolares entre 6 y 18 años, encontró una cortedad en torno al $25 \%$. Jordà en 1971 sobre una población de casi la misma edad, 6 y 14 años, encontró una menor incidencia de cortedad, siendo del 14-20\%. Santonja (1993) en su estudio sobre universitarios de Murcia encuentran un $27 \%$ de acortamientos bilaterales. Este mismo autor encuentra en jóvenes de 6-18 años una prevalencia de 49,2\% y $53,2 \%$, según se mida sobre la pierna derecha o izquierda respectivamente.

Estando muy generalizada esta disparidad de datos, lo que sí concuerda con nuestro estudio es que en las chicas se dan menos casos de cortedad que entre los chicos en ambos periodos temporales. Espiga (1992) indica que se da en un $20,9 \%$ de su muestra, siendo mayor en los chicos $29,4 \%$ frente a las chicas 7,9\%. Para López et al. (2008), en su estudio realizado sobre 64 piragüistas de $13,35 \pm 0,59$ años encuentran un $63,3 \%$ de cortedad para los chicos frente a un $22,7 \%$ para las chicas en su pierna derecha y un $27,2 \%$ para la izquierda. Santonja y Pastor (2003) informan que, al menos el $20 \%$ de los escolares presentan cortedad isquiosural. El mismo autor en otro estudio realizado junto a Ferrer (2000) encuentra un acortamiento entre los niños escolares del $28 \%$ y entre las niñas el $8,9 \%$. En nuestro estudio en el año 1986 encontramos un $0,39 \%$ de acortamiento entre las chicas frente a un $11,34 \%$ entre los chicos y en 2008 se incrementa el número de casos en ambos grupos, en las chicas a $6,83 \%$ y en los chicos al $32,04 \%$ (pero se incrementa mucho más entre los chicos).

\section{Conclusiones}

En el transcurso de 22 años que va desde 1986 a 2008, no solo se ha disminuido el nivel de flexibilidad isquiosural, sino que además se han incrementado en casi un $15 \%$ el número de casos de acortamiento, produciéndose este incremento tanto en las chicas como en los chicos.

\section{Referencias}

Bado, J. L. (1977). Dorso curvo. Montevideo: Artecolor.

Bado, J. L., Barros, P. C., Ruggiero, A., \& Navita, M. (1964). Análisis estadístico de la frecuencia de "retracción" de los isquiotibiales, estudiado en colectividades infantiles sanas y su relación con el dorso curvo. Anales de la Facultad de Medicina 49(1-2), 328-337. Montevideo: Universidad de la República.

Butler, R. W. \& Chir, M. (1995). The nature and significance of vertebral ostiochondrosis. Proceding of the Royal Society of Medicine, 48(11), 895-902.

Espiga, F. J. (1992). Estudio de prevalencia brevedad constitucional de la musculatura isquiotibial (Tesis doctoral). Universitat Autònoma de Barcelona, Barcelona.

Ferrer, V. (1998). Repercusión de la cortedad isquiosural sobre la pelvis y el raquis lumbar (Tesis doctoral). Universidad de Murcia, Murcia. 
Hass, C. J., Feigenbaum, M. S., \& Franklin, B. A. (2001). Prescription of Resistance Training for Healthy Populations. Sports Medicine, 31(14): 953-964. doi:10.2165/00007256-200131140-00001

Jordà, E. (1971). Brevedad de los isquiosurales. El síndrome de Bado en la gimnasia educativa y el deporte. Apunts. Medicina de l'Esport, 8(31), 123-124.

Kapanndji, I. A. (1977). Cuadernos de fisiología articular (pp. 24-25). Barcelona: Toray-Masson.

Kippers, V. \& Parker, A. W. (1987). Toe-touch test. Amesures of its validity. Physical Therapy, 67(11), 1680-1684.

Lambrinudi, C. (1934). Adolescent and senile kyphosis. British Medical Journal, 2(3852), 800-804. doi:10.1136/bmj.2.3852.800

Latorre, P. A. \& Hernández, J. A. (2003). Valoración de la condición física para la salud. Apunts. Educación Física y Deportes (73), 32-41.

López, P. A., Ferragut, C., Alacid, F., Yuste, J. L., \& García, A. (2008). Validez de los test dedos-planta y dedos-suelo para la valoración de la extensibilidad isquiosural en piragüistas de categoría infantil. Apunts. Medicina de l'Esport, 43(157), 24-9.

Micheli, L. J. (1979). Low back pain in the adolescent: Differential diagnosis. The American. Journal of Sports Medicine, 7(6), 362-364 doi: 10.1177/036354657900700613

Ministerio de Sanidad y Consumo, Ministerio de Educación, Política Social y Deporte. Programa Perseo: programa Piloto Escolar de referencia para la Salud y el Ejercicio contra la obesidad. Recupe- rado de http:www.perseo.aesan.msc.es/docs/docs/programa perseo/ material_divulgativo/Actividad_fisica_FINAL.pdf $>$

Moreno, B. \& Charro, A. (2007). Nutrición. Actividad física y prevención de la obesidad. Estrategia NAOS. Madrid: Panamericana.

Rodríguez, P. L., Santonja, F., Canteras, M., Delgado, M., Fernández, J., \& Balsalobre, J. (1999). Mejora de la extensibilidad isquiosural tras un programa escolar de estiramientos. Selección, 8(1), 35-41.

Santonja, F. (1993). Exploración clínica y radiográfica del raquis sagital. Sus correlaciones [Microficha]. Murcia: Secretariado de Publicaciones e Intercambio Científica, Universidad de Murcia.

Santonja, F. \& Pastor, A. (2003). Cortedad isquiosural y actitud cifótica lumbar. Selección, 12(3) 150-154.

Santonja, F., Andujar, P., \& Martínez, I. (1994). Ángulo lumbo-horizontal y valoración de repercusiones del síndrome de isquiotibiales corts. Apunts. Medicina de l'Esport. 31(120), 103-111.

Santonja, F., \& Ferrer, V. (2000). Síndrome de los isquiosurales cortos. En J. M. Arribas et al., Cirugía menor y procedimientos en medicina de familia. (pp. 793-803) Madrid: Jarpyo editores.

Santonja, F., Ferrer, V., \& Martínez, I. (1995). Exploración clínica del síndrome de isquiotibiales cortos. Selección, 4(2), 81-91.

Vidal M., Vidal T., \& Almela M. (2009). Valoración de la condición física en jóvenes medida en dos momentos temporales separados por 16 años. PubliCE Standard. Recuperado de http://www.sobreentrenamiento.com/PubliCE/Articulo.asp?ida $=1179$ 